\title{
Non-covalent graphene nanobuds from mono- and tripodal binding motifs
}

\author{
Marina Garrido, $\S^{\mathrm{a}}$ Joaquín Calbo, $\S^{\mathrm{b}}$ Laura Rodríguez-Pérez, ${ }^{\mathrm{a}}$ Juan Aragó, ${ }^{\mathrm{b}}$ Enrique Ortí, ${ }^{\mathrm{b}}$
} Má Ángeles Herranz ${ }^{* a}$ and Nazario Martín*ac

Graphene nanobuds were prepared via the non-covalent anchoring of $\mathrm{C}_{60}$-based molecules endowed with one or three pyrene units. TGA, FTIR, UV-Vis and TEM investigations confirmed the nanohybrids formation. For the two molecular derivatives, striking differences were determined in their interaction with graphene or carbon surfaces by Raman, cyclic voltammetry and molecular mechanics calculations, revealing the important role of pyrene adsorption in the electronic properties of the nanohybrids.

Extensive research efforts have been dedicated to carbonbased materials in the last decades. ${ }^{1}$ Especially, the combination of outstanding electronic, optical and mechanical properties of graphene has attracted considerable interest. ${ }^{2}$ Advances in its production by liquid phase dispersion and exfoliation of graphite have allowed its scalability and straightforward transfer to different substrates for many applications. $^{3}$

Graphene increasing availability is readily inspiring its chemical combination with different molecules and polymers in the search for novel nanomaterials with improved properties. ${ }^{4}$ One of the most interesting arrangements is that formed with fullerenes; in particular, the so-called graphene nanobuds arising from the covalent attachment of fullerene $\mathrm{C}_{60}$ to graphene. ${ }^{5}$ These species have shown an enhanced nonlinear optical response compared with the individual components, ${ }^{6}$ exhibit electron transfer from graphene to $C_{60}$ upon photoexcitation, ${ }^{7}$ and work as electrocatalysts toward the oxygen reduction reaction (ORR) showing an improved ORR activity with respect to pristine graphite. ${ }^{8}$

However, the creation of covalent bonds in the graphene structure disrupts the $\pi$-structure of the basal plane of graphene and causes the modification of its electronic properties. ${ }^{9}$ In contrast, non-covalent functionalization takes advantage of the molecular adsorption onto the graphene surface, and allows the preservation of the $\pi$-skeleton and the properties related to it. ${ }^{10}$

Here we report a new type of graphene nanobuds, in which covalently linked $\mathrm{C}_{60}$-pyrene conjugates (Fig. 1) are immobilized onto the surface of graphene via non-covalent forces. The use of pyrene derivatives is critical to ensure graphene dispersion and functionalization through directed $\pi-\pi$ interactions. ${ }^{11}$ Furthermore, the effect of the multivalent binding of tripodal receptors endowed with three pyrene units vs. the monopodal linkers has also been studied with the aim of modulating graphene electronic properties and establishing structure-property relationships in the obtained nanohybrids.

This journal is (C) The Royal Society of Chemistry 20xx

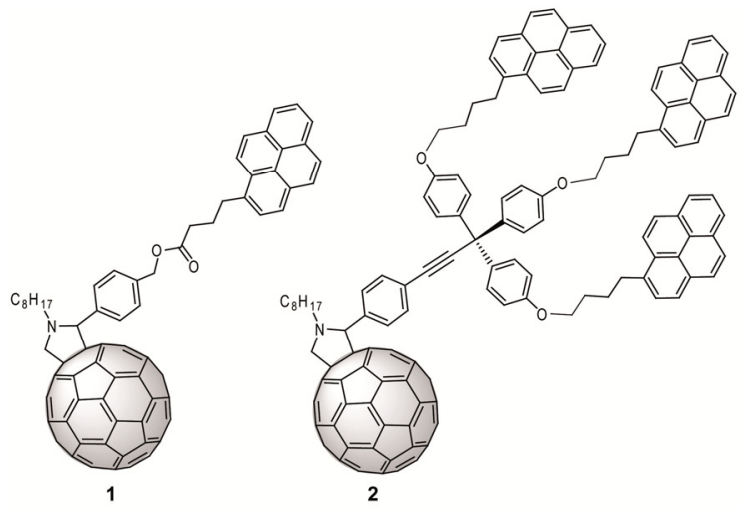

Fig. $1 C_{60}$ derivatives endowed with mono- (1) and tripodal (2) pyrene units.

The pyrene-fullerene dyads 1 and 2 (Fig. 1) were obtained with moderate yields (35-38\%) by a 1,3-dipolar cycloaddition reaction between the corresponding pyrene-based aldehydes (see $\mathrm{ESI}+$ for details) with $\mathrm{N}$-octylglycine and $\mathrm{C}_{60}$, following the procedure reported by Prato et al. ${ }^{12}$ Characterization was carried out by standard spectroscopic techniques. The pyrrolidine proton doublets and singlet are observed in ${ }^{1} \mathrm{H}$ NMR between 5.10 and 4.10 ppm for 1 , and between 4.95 and $3.85 \mathrm{ppm}$ for 2 . In ${ }^{13} \mathrm{C}-\mathrm{NMR}$, the signals for the $s p^{3}$ carbons of the pyrrolidine ring and for the $[6,6]$ junction of the $C_{60}$ framework are observed in the region between 84.4 and 65.9 ppm. The structure of $\mathbf{1}$ and $\mathbf{2}$ was unequivocally confirmed by exact MALDI-TOF-MS spectrometry.

In the case of $\mathbf{2}$, a self-assembly behaviour was suggested by concentration-dependent and variable-temperature ${ }^{1} \mathrm{H}$ NMR experiments. As the concentration increases (Fig. S1, $\mathrm{ESI}+$ ) or the temperature decreases (Fig. S2), most of the ${ }^{1} \mathrm{H}$ NMR signals broaden and shift upfield, which is in line with the formation of aggregates through $\pi-\pi$ interactions. ${ }^{13}$ ROESY and DOSY investigations further corroborated this hypothesis. Besides a number of intramolecular through-space coupling signals between the protons spatially close within the molecule, ROESY signals corresponding to intermolecular contacts between the protons of the pyrrolidine and the methylene units of the alkyl chains, as well as those of the aromatic protons of the benzene and pyrene units, were observed (Fig. S3). In DOSY experiments performed at two different concentrations $(2.5$ and $20 \mathrm{mM})$, the diffusion coefficient decreased from $4.09 \times 10^{6}$ to $3.03 \times 10^{6} \mathrm{~cm}^{2} / \mathrm{s}$, suggesting the presence of larger size species. The organization and morphology of derivatives $\mathbf{1}$ and $\mathbf{2}$ on surfaces was studied by AFM and confocal microscopy. Whereas for 1 the individual molecules coexist with small aggregates (Fig. S4 and S5), AFM images confirm the ability of $\mathbf{2}$ to self-assembly forming spherical aggregates of around 100 $\mathrm{nm}$ (Fig. 2 left). Confocal microscopy additionally shows that 
these aggregates exhibit blue fluorescence, characteristic of pyrene compounds, when excited at $405 \mathrm{~nm}$ (Fig. 2 right).
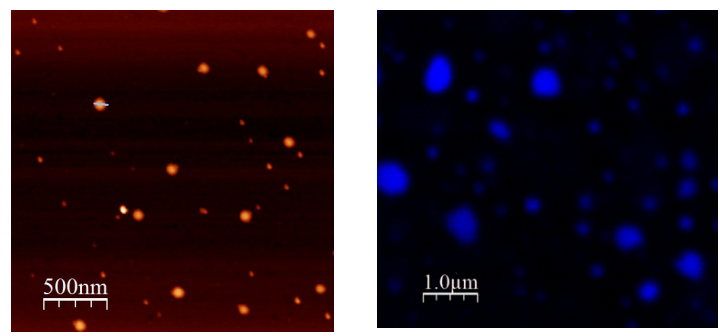

Fig. 2 AFM (left) and confocal microscopy (right) images of a drop-cast chloroform solution of 2. ( $\lambda_{\mathrm{exc}}=405 \mathrm{~nm}$ for the confocal microscopy).

To prepare the supramolecular complexes of $\mathbf{1}$ or $\mathbf{2}$ with graphene, the desired molecule was suspended in a graphene dispersion previously exfoliated in $\mathrm{N}$-methylpyrrolidone (NMP). ${ }^{14}$ The mixture was sonicated during 30 minutes and subsequently filtered and washed with dichloromethane to remove the excess of $\mathbf{1}$ or $\mathbf{2}$. The washings were repeated until the filtrate was transparent (see ESIt for specific details).

The supramolecular complexes were firstly investigated by thermogravimetric analysis (TGA) under inert atmosphere. The nanohybrids formed with $\mathbf{1}$ and $\mathbf{2}$ present a loss of weight compared with graphene of $2.82 \%$ and $11.18 \%$ at $600{ }^{\circ} \mathrm{C}$, respectively (Figure S6). At the same temperature, 1 reveals a loss of $30.29 \%$ and $\mathbf{2}$ of $44.95 \%$ (Figure S7). With these data in hand, adsorption ratios of a single molecule of 1 per 1020 carbon atoms of graphene and a single molecule of $\mathbf{2}$ per 508 atoms of graphene can be estimated. ${ }^{15}$

FTIR spectroscopy complementary confirmed the noncovalent functionalization of graphene. For the 1.graphene nanohybrid (Fig. 3), the graphene skeleton in-plane vibrations at $1583 \mathrm{~cm}^{-1}$, the vibrational band at $1736 \mathrm{~cm}^{-1}$ of the carbonyl group from the ester in $\mathbf{1}$ and the characteristic vibrational peak of $\mathrm{C}_{60}$ at $527 \mathrm{~cm}^{-1}$ corroborate the successful supramolecular modification of graphene.

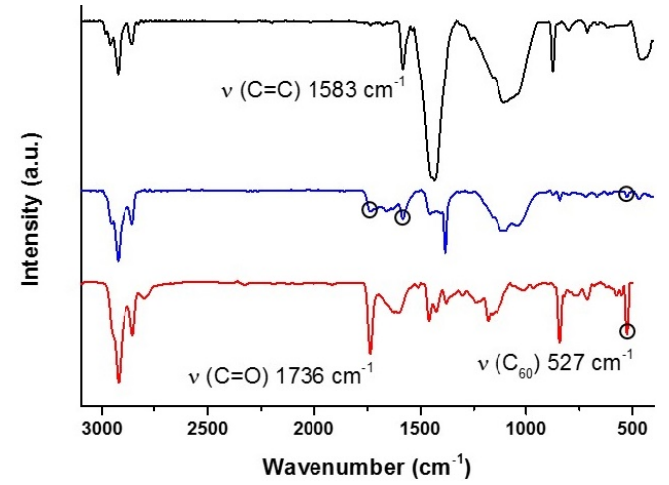

Fig. 3 FTIR spectra of graphene (black), 1.graphene nanohybrid (blue) and $\mathbf{1}$ (red).

In the UV-Vis spectra of the graphene nanohybrids (Fig. S8), the absorption bands below $350 \mathrm{~nm}$ corresponding to the pyrene moieties, together with the characteristic broad absorption tail of graphene that extends to the visible, are clearly observable. Furthermore, the interaction of graphene with $\mathbf{1}$ or $\mathbf{2}$ was investigated by UV-Vis titration experiments in solution (Figs. S9 and S10). In both cases, the presence of pseudo-isosbestic points validate the association between 1 or 2 and graphene. In fluorescence titration experiments, when increasing amounts of graphene are added to solutions of 1 and 2, a quenching of the pyrene fluorescence (ca. 15\%) is observed (Figs. S11 and S12), which additionally illustrates the interaction between both derivatives and graphene.

The morphology of the non-covalent nanohybrids was studied by TEM. Representative images obtained for 1.graphene are shown in Fig. 4. After the non-covalent modification, the graphene material is still formed by few layers and the re-aggregation is prevented by the presence of the pyrene- $\mathrm{C}_{60}$ molecules. When the images are obtained at 10 $\mathrm{nm}$ magnification, small round-shaped forms with diameters of around $1 \mathrm{~nm}$ are detected at the edges of the graphene, thus proving the presence of $C_{60}$ in the sample.
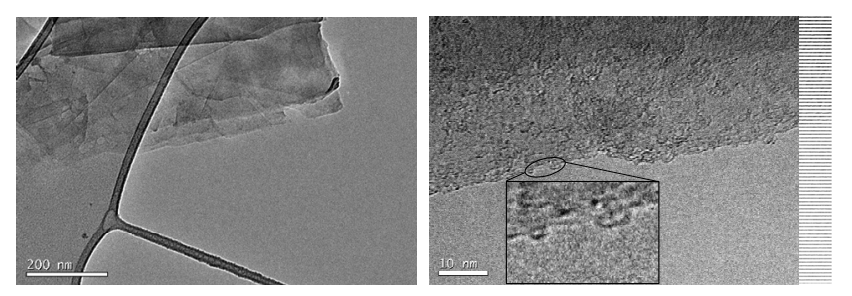

Fig. 4 TEM images obtained after the non-covalent functionalization of graphene with 1.

Raman spectroscopy was used to examine the structural and electronic characteristics of the obtained complexes (Fig. 5). As expected, the $I_{D} / I_{G}$ ratio of graphene does not increase after the non-covalent modification since graphene's structure was not altered in the process. For the $\mathrm{G}$ band, a shift to lower frequencies was surprisingly found for both complexes. Considering the electron-withdrawing ability of $\mathrm{C}_{60}$, the G-band wavenumber of graphene was expected to shift upwards due to the $p$-doping effect of $C_{60}$. These findings suggest that for both complexes the interaction that takes place through the pyrene moieties has an important impact on the electronic properties of graphene, as recently reported for related systems. ${ }^{15}$ The shift of the $\mathrm{G}$ band is higher for the $\mathbf{2}$.graphene complex, since the attachment to graphene via the pyrene "feet", acting as donor groups, causes a stronger electronic effect than in the monopodal 1.graphene system.

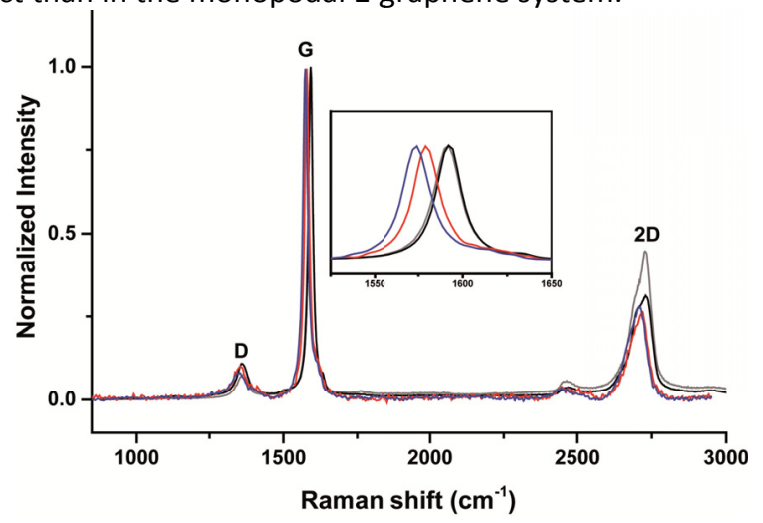

Fig. 5 Raman spectra $\left(\lambda_{\text {exc }}=532 \mathrm{~nm}\right.$ ) of graphite (grey), graphene (black) and graphene complexes with $\mathbf{1}$ (red) and $\mathbf{2}$ (blue). 
In order to further demonstrate the different binding behaviour to a carbon surface of the monopodal and tripodal systems, cyclic voltammograms were recorded onto the surface of a glassy carbon electrode, where compounds $\mathbf{1}$ and $\mathbf{2}$ were adsorbed upon consecutive cycles (Fig. S13). In the case of 1, all the redox processes are clearly observed as the number of scans increases and the adsorption of the molecule takes place. In contrast, for 2, the reductions associated with the $C_{60}$ unit disappear upon 10 consecutive scans. In this case, the adsorption of the pyrene units in a close proximity favours pyrene polymerization on the electrode, and the electrochemical response of the fullerene adduct is no longer observed.

To shed light into the non-covalent interactions that govern the self-assembly of $\mathbf{1}$ and $\mathbf{2}$ with graphene, molecular mechanics/molecular dynamics (MM/MD) calculations were performed using the general MM3 force field (see the ESIt for computational details). MD simulations were carried out for the nanohybrids of the two receptors with a graphene monolayer large enough to bear the bigger tripodal derivative, and were first performed in gas phase (298 K).

$\mathrm{MM} / \mathrm{MD}$ calculations predict that $\mathbf{1}$ favourably interacts with graphene through both the pyrene foot and the $\mathrm{C}_{60}$ moiety (Fig. S15). Interestingly, two well-differentiated regimes are found for 1 depending on the distance between the pyrene and $C_{60}$ units: regime $A$ involving folded structures with short pyrene- $C_{60}$ distances of around $9 \AA$ (Fig. S15, 5-7 ns), and regime $B$ characterized by extended conformations in which the two moieties are separated by more than $14 \AA$ (Fig. S15, > 7 ns). Both conformations are predicted with similar interaction energies. Fig. $6 a$ displays a representative example of the folded conformers (regime A) obtained during the dynamics.

In the case of 2, theoretical calculations predict a large recognition of the pyrene-based derivative to graphene by means of the three pyrene feet. The initial structure, described by an extended conformation with the three feet well separated from each other $(\sim 25 \AA)$ and the fullerene moiety lifted away from the graphene sheet (tilting angle of $\mathrm{C}_{60}$ with respect to the triple $\mathrm{C} \equiv \mathrm{C}$ bond $\theta=160-180^{\circ}$; Fig. S16c), rapidly evolves to more folded arrangements with pyrene-pyrene distances shorter than $20 \AA$ and $\theta=100-120^{\circ}$ (Fig. S16a-b). First, the fullerene moiety bends to interact with one pyrene foot (Fig. S16d). Then, the fullerene head is able to reach the graphene sheet and separates two neighbouring pyrene units at a fixed distance of $\sim 17 \AA$ (Fig. $6 b$ and Fig. S16e). This conformation is the most visited spatial arrangement of the tripodal nanobud along the simulation in gas phase.

Representative snapshots of the molecular dynamics were extracted for both supramolecular complexes and their geometries were optimized using the MM3 force field keeping the atoms of the graphene sheet frozen. The minimum-energy geometry obtained for the monopodal derivative 1 (Fig. 6a) indicates stabilizing $\pi-\pi$ interactions between pyrene and graphene in the 3.2-3.6 $\AA$ range, together with short $\mathrm{CH} \cdots \pi$ $(2.8-3.2 \AA)$ and $\mathrm{C}=0 \cdots \pi(3.4-3.6 \AA)$ contacts between the ester "leg" and graphene (Fig. S17a). Additionally, a short $\pi-\pi$ interaction between the fullerene ball and graphene is predicted around $3.0 \AA$, along with a large number of $\mathrm{CH} \cdots \pi$ contacts in the 2.8-3.1 $\AA$ range. Moving to 2, calculations predict short $\pi-\pi$ interactions in the 3.3-3.7 $\AA$ range between the three pyrene units and graphene (Fig. $6 \mathrm{~b}$ and S17b), as in the case of the monopodal derivative 1. During the dynamics, the fullerene head interacts with graphene through both the $\mathrm{C}_{60}$ ball with short contacts in the range of 2.9-3.1 $\AA$ and the long aliphatic chain with $\mathrm{CH} \cdots \pi$ interactions at $2.8-3.1 \AA$. The phenoxy legs also interact with the graphene sheet through $\mathrm{CH} \cdots \pi(2.8-3.0$ $\AA$ ) and $0 \cdots \pi(3.0 \AA$ $)$ contacts. Calculations therefore predict that both $\mathbf{1}$ and $\mathbf{2}$ are able to interact with graphene by the pyrene feet and the $\mathrm{C}_{60}$ unit. This agrees with the reduction peaks of $\mathrm{C}_{60}$ observed for $\mathbf{1}$ and $\mathbf{2}$ in the cyclic voltammograms recorded onto glassy carbon electrodes (see above).

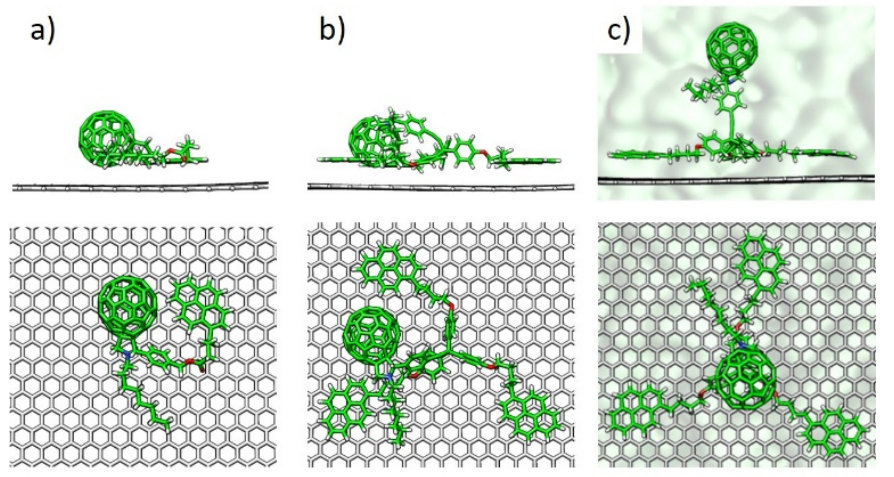

Fig. 6 Side and top views of the minimum-energy geometry calculated at the MM3 level for a representative structure of the supramolecular assembly of graphene with $\mathbf{1}$ (a) and $\mathbf{2}$ (b) in gas phase, and with $\mathbf{2}$ in the presence of the solvent (c).

Interaction energies $\left(E_{\text {int }}\right)$ were calculated at the MM3 level for the representative minimum-energy geometries described above for the monopodal and tripodal nanohybrids (Fig. 6a-b). Deformation energies were not considered. ${ }^{17} E_{\text {int }}$ of -72.3 and $129.1 \mathrm{kcal} / \mathrm{mol}$ were calculated for $\mathbf{1}$.graphene and $\mathbf{2}$.graphene, respectively. As a reference, the interaction energy of a pyrene molecule with graphene is computed to be $-23.4 \mathrm{kcal} / \mathrm{mol}$. Thus, the $E_{\text {int }}$ values calculated for both $\mathbf{1}$ and $\mathbf{2}$ are found much larger than expected from a simple pyrene-graphene recognition (for $2,-23.4 \times 3=-70.2 \mathrm{kcal} / \mathrm{mol}$ ). This is due to the presence of other supramolecular interactions originated from the $\mathrm{C}_{60}$ counterpart and the chains linking $\mathrm{C}_{60}$ with pyrene-

$\mathrm{MM} / \mathrm{MD}$ calculations were also performed including explicitly solvent effects using the NPT ensemble (see the ESI ${ }^{\dagger}$ for a full description). Simulations for monopod 1 led to similar results as obtained in gas phase (Fig. S18). Otherwise, calculations for tripod $\mathbf{2}$ in solution demonstrated that the folded conformation in which the $\mathrm{C}_{60}$ ball interacts with graphene (Fig. 6b) evolves to more erected dispositions where this interaction is no longer present (Fig. 6c). The upright conformer is calculated with an $E_{\text {int }}$ of $-107.4 \mathrm{kcal} / \mathrm{mol}$, suggesting that the $\mathrm{C}_{60}$-graphene interaction is around -20 $\mathrm{kcal} / \mathrm{mol}$. This interaction is however not strong enough to preserve the fullerene-graphene assembly in the presence of the solvent, as MM/MD simulations confirm (Fig. S22). The decoration with pyrene units in $\mathbf{1}$ and $\mathbf{2}$ is therefore key for building stable non-covalent graphene nanobuds. 
In summary, by using a facile solution methodology, we successfully formed non-covalent graphene nanobuds by the combination of exfoliated graphene and mono- or tripodal pyrene units linked covalently to $C_{60}$. The formation of the nanohybrids was confirmed by TGA, FTIR, Raman, UV-Vis and TEM characterization. The supramolecular forces that contributed to stabilize the nanostructures were investigated by $\mathrm{MM} / \mathrm{MD}$ calculations and the most plausible interacting conformations helped in rationalizing the experimental findings, which point to a multivalent effect of the pyrene units in the tripodal systems. Further investigations are being carried out with extended series of pyrene-based receptors of graphene, incorporating electroactive units different from $\mathrm{C}_{60}$, in order to fine-tune and control its electronic properties by chemical modification.

Financial support from the European Research Council (ERC320441-Chirallcarbon), MINECO of Spain (CTQ2014-52045-R, CTQ2015-71154-P, CTQ2015-71936-REDT, Unidad de Excelencia María de Maeztu MDM-2015-0538), Comunidad de Madrid (S2013/MIT-2841), Generalitat Valenciana (PROMETEO/2016/ 135) and European FEDER funds (CTQ2015-71154-P) is acknowledged.

\section{Notes and references}

1 M. Antonietti and K. Mullen, Chemical Synthesis and Applications of Graphene and Carbon Materials, Wiley-VCH, Weinheim, 2016.

2 (a) Y. Zhu, S. Murali, W. Cai, X. Li, J. W. Suk, R. Potts and R. S Ruoff, Adv. Mater., 2010, 22, 3906; (b) A. K. Geim, Science, 2009, 324, 1530.

3 (a) A. Ciesielski and P. Samori, Adv. Mater., 2016, 28, 6030; (b) D. Parviz, F. Irin, S. A. Shah, S. Das, C. B. Sweeney and M. J. Green, Adv. Mater., 2016, 28, 8796; (c) K. S. Mali, J. Greenwood, J. Adisoejoso, R. Phillipson and S. De Feyter, Nanoscale, 2015, 7, 1566

4 (a) G. Bottari, M. A. Herranz, L. Wibmer, M. Volland, L. Rodríguez-Pérez, D. M. Guldi, A. Hirsch, N. Martín, F. D'Souza, T. Torres, Chem. Soc. Rev., 2017, 46, 4464; (b) S. Eigler and A. Hirsch, Angew. Chem. Int. Ed., 2014, 53, 7720; (c) L. RodríguezPérez, M. A. Herranz and N. Martín, Chem. Commun., 2013, 49, 3721; (d) V. Georgakilas, M. Otyepka, A. B. Bourlinos, V. Chandra, N. Kim, K. C. Kemp, P. Hobza, R. Zboril and K. S. Kim, Chem. Rev., 2012, 112, 6156

5 (a) V. Georgakilas, A. B. Bourlinos, , E. Ntararas, A. Ibraliu, D. Gournis, K. Dimos, A. Kouloumpis and R. Zboril, Carbon, 2016, 110, 51; (b) D. García, L. Rodríguez-Pérez, M. A. Herranz, D.
Peña, E. Guitián, S. Bailey, Q. Al-Galiby, M. Noori, C. Lambert, D. Pérez and N. Martín, Chem. Commun., 2016, 52, 6677.

6 X. Zhang, Z. Zhi, Y. Huang, X. Wan, J. Tian, Y. Ma and Y. Chen, J. Nanosci. Nanotech., 2009, 9, 5752.

7 M. Barrejón, M. Vizuete, M. J. Gómez-Escalonilla, J. L. Fierro, I. Berianga, F. Zamora, G. Abellán, P. Atienzar, J.-F. Nierengarten, H. Garcia and F. Langa, Chem. Commun., 2014, 50, 9053.

8 J. Guan, X. Chen, T. Wei, F. Liu, S. Wang, Q. Yang, Y. Lu and S. Yang, J. Mater. Chem. A, 2015, 3, 4139.

9 D. R. Dreyer, S. Park, C. W. Bielawski and R. S. Ruoff, Chem. Soc. Rev., 2010, 39, 228.

10 (a) A. Ciesielski, S. Haar, M. El Gemayel, H. Yang, J. Clough, G. Melinte, M. Gobbi, E. Orgiu, M. V. Nardi, G. Ligorio, V. Palermo, N. Koch, O. Ersen, C. Casiraghi and P. Samori, Angew. Chem. Int. Ed. 2014, 53, 10355; (b) P. Bai, J. Kao, J.-H. Chen, W. Mickelson, A. Zettl and T. Xu, Nanoscale, 2014, 6, 4503.

11 (a) J. A. Mann and W. R. Dichtel, Phys. Chem. Lett., 2013, 4, 2649; (b) D. Parviz, S. Das, H. S. T. Ahmed, F. Irin, S. Bhattacharia, M. J. Green, ACS Nano, 2012, 6, 8857; (c) J. A. Mann, J. Rodríguez-López, H. D. Abruña, W. R. Dichtel, J. Am. Chem. Soc., 2011, 133, 17614.

12 (a) M. Prato, M. Maggini, Acc. Chem. Res., 1998, 31, 519; (b) M. Maggini, G. Scorrano, M. Prato, J. Am. Chem. Soc., 1993, 115, 9798.

13 (a) J. S. Valera, J. Calbo, R. Gómez, E. Ortí and L. Sánchez, Chem. Commun., 2015, 51, 10142; (b) G. Fernández, E. M. Pérez, L. Sánchez and N. Martín, Angew. Chem. Int. Ed., 2008, 47, 1094; (c) E. M. Pérez, N. Martín, Chem. Soc. Rev., 2015, 44, 6425.

14 The graphene material was obtained through graphite exfoliation following Coleman's procedure toward few-layered graphene suspensions: Y. Hernandez, V. Nicolosi, M. Lotya, F. M. Blighe, Z. Sun, S. De, I. T. McGovern, B. Holland, M. Byrne, Y. K. Gun'Ko, J. J. Boland, P. Niraj, G. Duesberg, S. Krishnamurthy, R. Goodhue, J. Hutchison, V. Scardaci, A. C. Ferrari and J. N. Coleman, Nat. Nanotechnol., 2008, 3, 563. To simplify the discussions we will keep referring from here on to the suspensions as graphene.

15 For details about the calculations see: M. A. Herranz, C. Ehli, S. Campidelli, M. Gutiérrez, G. L. Hug, K. Ohkubo, S. Fukuzumi, M. Prato, N. Martín, D. M. Guldi, J. Am. Chem. Soc., 2008, 130, 66.

16 (a) S. Qu, M. Li, L. Xie, X. Huang, J. Yang, N. Wang and S. Yang, ACS Nano, 2013, 7, 4070; (b) K. Dirian, M. A. Herranz, G. Katsukis, J. Malig, L. Rodríguez-Pérez, C. Romero-Nieto, V. Strauss, N. Martín and D. M. Guldi, Chem. Sci., 2013, 4, 4335.

17 Density functional theory calculations at the B3LYP/6-31G** level of theory demonstrated that the deformation energy provoked by the tilting of $\mathrm{C}_{60}$ in the tripodal host/graphene recognition amounts to only $7 \mathrm{kcal} / \mathrm{mol}$. 


\section{Table of contents entry}

Dispersion forces govern the interaction of graphene with mono- and tripodal pyrene-[60]fullerene derivatives and direct the formation of graphene nanobuds

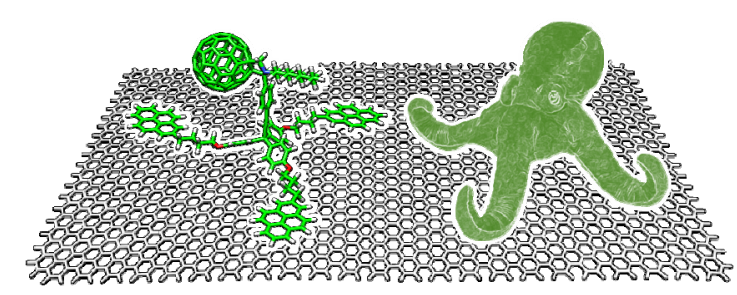

